\title{
A NOTE ON THE FOURIER COEFFICIENTS OF HALF-INTEGRAL WEIGHT MODULAR FORMS
}

\author{
NARASIMHA KUMAR AND SOMA PURKAIT
}

\begin{abstract}
In this note, we show that the algebraicity of the Fourier coefficients of half-integral weight modular forms can be determined by checking the algebraicity of the first few of them. We also give a necessary and sufficient condition for a half-integral weight modular form to be in Kohnen's +-subspace by considering only finitely many terms.
\end{abstract}

\section{INTRODUCTION}

In the theory of modular forms, it is of fundamental interest to understand the algebraicity of the Fourier coefficients of modular forms. It is well-known that a normalized Hecke eigenform of integral weight has algebraic Fourier coefficients, since the Hecke eigenvalues coincide with the Fourier coefficients. Moreover, there exists a number field containing all these Fourier coefficients. However, for half-integral weight modular forms, the authors are not aware of any such results.

In this note, we show that if the Fourier coefficients of a half-integral weight modular form are algebraic up to Sturm's bound (for half-integral weight modular forms), which is specified in terms of the level and weight of the corresponding modular form, then so are all others (cf. Theorem 3.2 in the text).

In his remarkable work [3], Kohnen defines the +-subspace (new) and proves that it is isomorphic to a space of newforms of integral weight via certain linear combination of Shimura correspondences. In the last section, we give a necessary and sufficient condition for a half-integral weight modular form to be in Kohnen's +-subspace by checking the vanishing condition up to Sturm's bound.

\section{Multiplication by Theta series}

Let $k>1$ be an odd integer. Let $f$ be a half-integral weight modular form of weight $k / 2$, level $N$ with $4 \mid N$, and an even Dirichlet character $\chi$. In particular, $f \in M_{k / 2}\left(\Gamma_{1}(N)\right)$, therefore $f$ has a Fourier expansion of the form $f(z)=\sum_{n=0}^{\infty} a_{f}(n) q^{n}$, where $q=e^{2 \pi i z}$. Let $\Theta(z)=\sum_{n=0}^{\infty} b_{\Theta}(n) q^{n^{2}}$ with $b_{\Theta}(0)=1, b_{\Theta}(n)=2$ for all $n \geq 1$.

In this section, we show that the Fourier coefficients of $f$ are algebraic if the Fourier coefficients of $f \Theta$ are algebraic.

By [9], we know that $\Theta \in M_{1 / 2}\left(4, \chi_{\text {triv }}\right)$, where $\chi_{\text {triv }}$ stands for the trivial Dirichlet character. Define

$$
g:=f \Theta .
$$

If $f \in M_{k / 2}(N, \chi)$, then it is easy to see that $g \in M_{\frac{k+1}{2}}\left(N, \chi \cdot \chi_{-1}^{\frac{k+1}{2}}\right)$, where $\chi_{-1}$ is the non-trivial Dirichlet character modulo 4.

2010 Mathematics Subject Classification. Primary 11F30, 11F37; Secondary 11F11.

Key words and phrases. Modular forms, half-integral weight, algebraicity, Sturm's bound. 
Suppose $g(z)$ has the $q$-expansion given by $g(z)=\sum_{n=0}^{\infty} c(n) q^{n}$. Since $g$ is the product of $f$ and $\Theta$, we see that for all $n \geq 0$, we have

$$
c(n)=\sum_{x+y^{2}=n} a_{f}(x) b_{\Theta}(y) .
$$

In general, the product of two eigenforms need not be an eigenform, in particular $f \Theta$ need not be an eigenform. Hence, one cannot conclude that the Fourier coefficients of $f \Theta$ generate a number field. However, if we assume that Fourier coefficients of $f \Theta$ generate a number field, then by a simple induction argument, we can show that the Fourier coefficients of $f$ also generate the same number field.

Instead of proving the above claim for $f$ and $\Theta$, we prove it for the product of two general half-integral weight modular forms.

Proposition 2.1. Let $f(z)=\sum_{n=n_{0}}^{\infty} a_{n} q^{n} \in M_{k_{1} / 2}\left(N_{1}, \chi_{1}\right), h(z)=\sum_{n=n_{1}}^{\infty} b_{n} q^{n} \in$ $M_{k_{2} / 2}\left(N_{2}, \chi_{2}\right)$ be two modular forms such that $a_{n_{0}} \neq 0, b_{n_{1}} \neq 0$. Suppose that the Fourier coefficients of the product fh belong to a number field $K$. If $a_{i} \in K$ for all $i \geq n_{0}$, then $b_{i} \in K$ for all $i \geq n_{1}$.

Proof. We prove the proposition by induction. Suppose

$$
f h=\sum_{n=n_{0}+n_{1}}^{\infty} c_{n} q^{n}
$$

is the $q$-expansion of $f h$.

Look at the first non-zero coefficient of the product $f h$, i.e., $n_{0}+n_{1}$-th term of $f h$, which is $c_{n_{0}+n_{1}}=a_{n_{0}} b_{n_{1}} \in K$. Since $a_{n_{0}} \neq 0$, we get that $b_{n_{1}}$ is a non-zero element of $K$.

Now, look at the $n_{0}+n_{1}+1$-th term of the product $f h$, which is $c_{n_{0}+n_{1}+1}=a_{n_{0}} b_{n_{1}+1}+$ $a_{n_{0}+1} b_{n_{1}} \in K$. Since $a_{n_{0}}, a_{n_{0}+1}, b_{n_{1}} \in K$, we get that $b_{n_{1}+1} \in K$.

Now, we assume that $b_{n_{1}}, b_{n_{1}+1}, \ldots, b_{n_{1}+r-1} \in K$ and show that $b_{n_{1}+r} \in K$. We can write

$$
c_{n_{0}+n_{1}+r}=a_{n_{0}} b_{n_{1}+r}+a_{n_{0}+1} b_{n_{1}+r-1}+\ldots+a_{n_{0}+r} b_{n_{1}} \in K .
$$

Since $b_{n_{1}}, b_{n_{1}+1}, \ldots, b_{n_{1}+r-1} \in K$ and $a_{n_{0}}, a_{n_{0}+1}, \ldots, a_{n_{0}+r} \in K$, we see that $b_{n_{1}+r}$ also belongs to $K$. This proves the proposition.

Observe that the above proof also works for any two general Fourier $q$-expansions. Now, coming back to the pair $(f, \Theta)$, we have the following:

Corollary 2.2. If the coefficients $c_{n}(n \in \mathbb{N})$ of $g=f \Theta$ belong to a number field $K$, then the Fourier coefficients $a_{f}(n) \in K$ for all $n$.

We shall illustrate the above proposition with an example.

Example 1. Take $k=7, N=8$. Using MAGMA [2], we see that $S_{7 / 2}\left(8, \chi_{\text {triv }}\right)$ is onedimensional and is spanned by

$$
f=q-2 q^{2}-4 q^{5}+12 q^{6}-3 q^{9}-20 q^{10}+O\left(q^{12}\right) .
$$

Now consider the integral weight modular form $g:=f \Theta$. This is an element of $S_{4}\left(8, \chi_{\text {triv }}\right)$, which is of dimension 1. Moreover, $S_{4}^{\text {new }}\left(8, \chi_{\text {triv }}\right)$ is also of dimension 1 . By comparing the Fourier coefficient of $q$ in $f \Theta$, we see that

$$
S_{4}^{\text {new }}\left(8, \chi_{\text {triv }}\right)=\langle g\rangle \text {. }
$$

By Proposition 2.1, we see that the Fourier coefficients of $f$ generate a number field.

Remark 2.3. The same argument go through, if you replace $\Theta$ by $\Theta(\psi, 0, z)$ for $\psi$ an even Dirichlet character, where $\Theta(\psi, 0, z)=\sum_{n=0}^{\infty} \psi(n) b_{\Theta}(n)$. 
Let $d$ be a positive integer and $V(d)$ be the usual shift operator. If the Fourier coefficients of $f(z) \cdot V(d) \Theta(\psi, 0, z)$ belongs to a number field $K$, then so are the Fourier coefficients of $f(z)$.

\section{MAIN RESUlT}

In this section, we show that if the Fourier coefficients of a half-integral weight modular form are algebraic up to Sturm's bound (for half-integral weight modular forms), which is specified in terms of the level and weight of the corresponding modular form, then so are all others.

Let $k, N$ be positive integers with $k$ odd and $4 \mid N$. Let $\chi$ be an even Dirichlet character of modulus $N$. In his thesis Basmaji [1] gave an algorithm for computing a basis for the space of half-integral weight modular forms of level divisible by 16 . The main idea of the algorithm is to use theta series $\Theta=\sum_{n=-\infty}^{\infty} q^{n^{2}}, \Theta_{1}=\frac{\Theta-V(4) \Theta}{2}$ and the following embedding,

$$
\varphi: S_{k / 2}(N, \chi) \rightarrow S \times S, \quad f \mapsto\left(f \Theta, f \Theta_{1}\right),
$$

where $S=S_{\frac{k+1}{2}}\left(N, \chi \cdot \chi_{-1}^{\frac{k+1}{2}}\right)$. This idea was later generalized by Steve Donnelly for levels divisible by 4 by using different theta-multipliers [2].

We will be requiring the following lemma, which is an analogue of Sturm's Theorem [10, page 276] for half-integral weight modular forms.

Lemma 3.1. Let $f=\sum_{n=0}^{\infty} a_{f}(n) q^{n} \in M_{k / 2}(N, \chi)$ with $k$ odd. If $a_{f}(n)=0$ for $n \leq$ $\frac{k}{24}\left[\mathrm{SL}_{2}(\mathbb{Z}): \Gamma_{0}(N)\right]$, then $f=0$.

Proof. Set $B=\frac{k}{24}\left[\mathrm{SL}_{2}(\mathbb{Z}): \Gamma_{0}(N)\right]$. Since $a_{f}(n)=0$ for $n \leq B$, the Fourier expansion of $f$ at $\infty$ can be written as

$$
f=q^{B+1}\left(a_{f}(B+1)+a_{f}(B+2) q+\cdots\right) .
$$

Let $s$ be the order of the Dirichlet character $\chi$. Then, it is easy to see that $f^{4 s} \in$ $M_{2 k s}\left(\Gamma_{0}(N), \chi_{\text {triv }}\right)$ is an integral weight modular form. Clearly, the Fourier expansion of $f^{4 s}$ at $\infty$ looks like

$$
f^{4 s}=q^{4 s(B+1)}\left(\sum_{n=0}^{\infty} c_{n} q^{n}\right),
$$

where $c_{n}$ is in terms of $a_{f}(i)$ for $i \leq n$.

Since the Fourier coefficients of $f^{4 s}$ are zero up to $4 s B=\frac{2 k s}{12}\left[\mathrm{SL}_{2}(\mathbb{Z}): \Gamma_{0}(N)\right]$, by applying Sturm's theorem [10, page 276] to $f^{4 s}$, we get that $f^{4 s}=0$. This implies that $f=0$, which proves the lemma.

Our results are in the flavor of Sturm's work [10] on determining modular forms by checking congruences up to a finite number. Set $B_{k}(N):=\frac{k}{24} \cdot\left[\mathrm{SL}_{2}(\mathbb{Z}): \Gamma_{0}(N)\right]$.

Theorem 3.2. Let $f=\sum_{n=1}^{\infty} a_{f}(n) q^{n} \in S_{k / 2}(N, \chi)$ be a non-zero half-integral weight modular form. Suppose $a_{f}(m)$ is algebraic for all $1 \leq m \leq B_{k}(N)$. Then all Fourier coefficients are algebraic. Moreover, there exists a number field $K_{f}$ such that $a_{f}(n) \in K_{f}$ for all $n$.

Proof. By Basmaji's algorithm [1], we can construct a basis for the space of cusp forms $S_{k / 2}(N, \chi)$ such that the basis elements have Fourier coefficients defined over the number field generated by $\chi$. Let $f_{1}, f_{2}, \ldots, f_{r}$ denote such a basis of $S_{k / 2}(N, \chi)$. For each $i$, 
suppose $f_{i}:=\sum_{n=1}^{\infty} a_{i}(n) q^{n}$ is the $q$-expansion. Write $f=\sum_{i=1}^{r} \lambda_{i} f_{i}$ where $\lambda_{i} \in \mathbb{C}$. Hence, we have the following system of linear equations given by

$$
\left[\begin{array}{cccc}
a_{1}(1) & a_{2}(1) & \cdots & a_{r}(1) \\
\vdots & \vdots & \cdots & \vdots \\
a_{1}(m) & a_{2}(m) & \cdots & a_{r}(m) \\
\vdots & \vdots & \cdots & \vdots \\
a_{1}(n) & a_{2}(n) & \cdots & a_{r}(n) \\
\vdots & \vdots & \cdots & \vdots
\end{array}\right]\left[\begin{array}{c}
\lambda_{1} \\
\lambda_{2} \\
\vdots \\
\lambda_{r}
\end{array}\right]=\left[\begin{array}{c}
a_{f}(1) \\
\vdots \\
a_{f}(m) \\
\vdots \\
a_{f}(n) \\
\vdots
\end{array}\right]
$$

For simplicity, let $B$ denote $B_{k}(N)$. Now from the above matrix, we consider the first $B$ rows to form the following $B \times r$-matrix, where $B \geq r$ :

$$
A:=\left[\begin{array}{cccc}
a_{1}(1) & a_{2}(1) & \cdots & a_{r}(1) \\
\vdots & \vdots & \cdots & \vdots \\
a_{1}(m) & a_{2}(m) & \cdots & a_{r}(m) \\
\vdots & \vdots & \cdots & \vdots \\
a_{1}(B) & a_{2}(B) & \cdots & a_{r}(B)
\end{array}\right]
$$

We want to show that the rank of $A$ is $r$. Suppose not. Then, there exists $\alpha_{1}, \ldots, \alpha_{r} \in \mathbb{C}$, not all zero, such that

$$
\sum_{i=1}^{r} \alpha_{i} a_{i}(j)=0
$$

for each $j=1, \ldots, B$. Now, consider the half-integral weight modular form

$$
h:=\alpha_{1} f_{1}+\alpha_{2} f_{2}+\ldots+\alpha_{r} f_{r} .
$$

Now, by (3.3), the first $B$ coefficients of $h$ are zero. By Lemma 3.1, we see that the modular form $h$ is identically zero. Therefore, we have

$$
\alpha_{1} f_{1}+\alpha_{2} f_{2}+\ldots+\alpha_{r} f_{r}=0 .
$$

Since $f_{1}, \ldots, f_{r}$ are linearly independent, we see that all $\alpha_{i}$ 's have to be zero, which is a contradiction. Therefore, the matrix $A$ has rank $r$. Let $C$ be the $r \times r$ submatrix of $A$ with full rank $r$. Now, consider the following system of linear equations (formed from the (3.1))

$$
C\left[\begin{array}{c}
\lambda_{1} \\
\lambda_{2} \\
\vdots \\
\lambda_{r}
\end{array}\right]=\left[\begin{array}{c}
a_{f}\left(i_{1}\right) \\
a_{f}\left(i_{2}\right) \\
\vdots \\
a_{f}\left(i_{r}\right)
\end{array}\right]
$$

for some distinct $1 \leq i_{1}<i_{2}<\cdots<i_{r} \leq B$. Since $C$ is invertible, we see that $\lambda_{i}$ 's can be expressed as an algebraic linear combination of $a_{f}\left(i_{j}\right)$ for $j=1, \ldots, r$. Therefore, $\lambda_{i}$ 's are algebraic, hence all the Fourier coefficients of $f$ are algebraic.

Now, take $K_{f}$ to the number field generated by $\lambda_{1}, \ldots, \lambda_{n}$ and the values of $\chi$. Since $f=\sum_{i=1}^{n} \lambda_{i} f_{i}$, we see that $a_{f}(n) \in K_{f}$ for all $n$.

Remark 3.3. Using the same method one can see that the above theorem also holds for integral weight cusp forms for any level and nebentypus.

One could also use the idea of multiplying the half-integral weight modular form $f$ of any level and nebentypus by theta series $\Theta$ (as in Section 2) and argue in the integral weight case setting (cf. [5. Lemma 3.1]). In this approach, one may have to check the algebraicity of the Fourier coefficients of $f \Theta$ up to $\frac{k+1}{24}\left[\mathrm{SL}_{2}(\mathbb{Z}): \Gamma_{0}(N)\right]$ to conclude that all the other coefficients of $f \Theta$ are algebraic, and hence the coefficients of $f$ as well. However, if we 
work completely in the half-integral weight setting, it is sufficient to check the algebraicity of the Fourier coefficients of $f$ up to $B:=\frac{k}{24}\left[\mathrm{SL}_{2}(\mathbb{Z}): \Gamma_{0}(N)\right]$ (in particular, this implies that only the first $B$ coefficients of $f \Theta$ are algebraic). Hence, we chose to work in the half-integral weight setting which gives a bit better bound.

We predict that for an eigenform $f$ such a bound for the algebraicity can be sharpened by using Waldspurger's results [11]. We hope to come back to this in the future.

\section{When $N / 4$ IS ODD AND SQUARE-FREE}

Let $k, N$ be positive integers with $k \geq 3$ odd and $4 \mid N$. Let $\chi$ be an even quadratic Dirichlet character of modulus $N$.

Let $F=\sum_{n=1}^{\infty} A(n) q^{n}$ be a newform of weight $k-1$, level $N / 4$ odd and square-free with trivial nebentypus. Let $f$ be a non-zero element of $S_{k / 2}(N, \chi, F)$ (for the definition, see 4.11). In this case, checking the algebraicity of the Fourier coefficients of $f$ becomes practically effective.

4.1. An algebraic basis for $S_{k / 2}(N, \chi, F)$ : In this section, we shall recall some basic definitions and results.

Let $S_{k / 2}^{\prime}(N, \chi)$ be the orthogonal complement of the subspace of $S_{k / 2}(N, \chi)$ spanned by single-variable theta series with respect to the Petersson inner product. Note that for $k \geq 5$, we have $S_{k / 2}^{\prime}(N, \chi)=S_{k / 2}(N, \chi)$.

Let $N^{\prime}=N / 2$. For $M \mid N^{\prime}$ such that $\operatorname{Cond}\left(\chi^{2}\right) \mid M$ and $F \in S_{k-1}^{\text {new }}\left(M, \chi^{2}\right)$, Shimura defines

$$
S_{k / 2}(N, \chi, F)=\left\{f \in S_{k / 2}^{\prime}(N, \chi): T_{p^{2}}(f)=\lambda_{p}^{F} f \text { for almost all } p \nmid N\right\} ;
$$

here $T_{p}(F)=\lambda_{p}^{F} F$. In [6, Corollary 5.2] the second author gives an algorithm for computing these subspaces. We will need the following proposition in the next section.

Proposition 4.1. Let $F$ be a newform in $S_{k-1}^{\text {new }}\left(M, \chi^{2}\right)$ with level $M$ dividing $N^{\prime}$. Then there exists a basis of $S_{k / 2}(N, \chi, F)$ defined over the number field generated by the Fourier coefficients of $F$ and $\chi$.

Proof. By Basmaji's algorithm [1], one can construct a basis for the space of cusp forms $S_{k / 2}(N, \chi)$ defined over the number field generated by $\chi$. Let $f_{1}, f_{2}, \ldots f_{r}$ be such a basis and let $f_{i}=\sum_{n=1}^{\infty} a_{i}(n) q^{n}$. Let $p_{1}<p_{2}<\cdots<p_{s}$ be the primes chosen as in [6. Corollary 5.2]. Let $T_{p_{j}^{2}} f_{i}=\sum_{n=1}^{\infty} b_{i, j}(n) q^{n}$ for $1 \leq i \leq r$ and $1 \leq j \leq s$. By the same corollary to construct a basis for $S_{k / 2}(N, \chi, F)$, we need to determine a basis of the solution space for the simultaneous homogeneous system of linear equations given by

$$
\left[\begin{array}{cccc}
\vdots & \vdots & \ldots & \vdots \\
b_{1,1}(n)-\lambda_{p_{1}}^{F} a_{1}(n) & b_{2,1}(n)-\lambda_{p_{1}}^{F} a_{2}(n) & \ldots & b_{r, 1}(n)-\lambda_{p_{1}}^{F} a_{r}(n) \\
\vdots & \vdots & \ldots & \vdots \\
b_{1, s}(n)-\lambda_{p_{s}}^{F} a_{1}(n) & b_{2, s}(n)-\lambda_{p_{s}}^{F} a_{2}(n) & \ldots & b_{r, s}(n)-\lambda_{p_{s}}^{F} a_{r}(n) \\
\vdots & \vdots & \ldots & \vdots
\end{array}\right]\left[\begin{array}{c}
x_{1} \\
x_{2} \\
\vdots \\
x_{r}
\end{array}\right]=\left[\begin{array}{c}
\vdots \\
0 \\
\vdots \\
0 \\
\vdots
\end{array}\right] .
$$

Since the matrix of the system is defined over number field generated by $F$ and $\chi$, the proposition clearly follows.

4.2. On determination of the algebraicity. Let $F$ be a newform of weight $k-1$ and level $N / 4$ odd and square-free with trivial nebentypus. Let $S_{k / 2}^{+}(N, \chi)$ denote Kohnen's +-subspace of $S_{k / 2}(N, \chi)$ consisting of modular forms $f=\sum_{n=1}^{\infty} a_{f}(n) q^{n}$ with $a_{f}(n)=0$ for $n \equiv 2,(-1)^{\frac{k+1}{2}}(\bmod 4)$. Set $S_{k / 2}^{+}(N, \chi, F)=S_{k / 2}^{+}(N, \chi) \cap S_{k / 2}(N, \chi, F)$. 
By Waldspurger [11, Théorème 1], we know that $S_{k / 2}(N, \chi, F)$ is 2-dimensional as $N / 4$ is odd and square-free. By Proposition 4.1, we know that there exists a basis $f_{1}, f_{2}$ of $S_{k / 2}(N, \chi, F)$, which are defined over the field generated by the Fourier coefficients of $F$ and $\chi$. By [3, Theorem 2], Kohnen's +-subspace $S_{k / 2}^{+}(N, \chi, F)$ is 1-dimensional. Suppose that $S_{k / 2}^{+}(N, \chi, F)=\left\langle f_{K}\right\rangle$.

Proposition 4.2. The Fourier coefficients of $f_{K}$ are algebraic up to a normalization.

Proof. Let $f_{K}(z)=\sum_{n=1}^{\infty} a_{K}(n) q^{n}$. Kohnen [3] gives the following isomorphism

$$
\mathcal{S}: S_{k / 2}^{+, \text {new }}(N, \chi) \stackrel{\sim}{\longrightarrow} S_{k-1}^{\text {new }}(N / 4),
$$

where $\mathcal{S}$ is a certain linear combination of Shimura correspondences. Hence there exists a unique $g \in S_{k / 2}^{+, \text {new }}(N, \chi)$ such that $\mathcal{S}(g)=F$. Since the Shimura correspondence commutes with Hecke operators for all primes $p$ [7, we get

$$
\mathcal{S}\left(T_{p^{2}} g\right)=T_{p}(\mathcal{S} g)=T_{p}(F)=\lambda_{p}^{F} F=\mathcal{S}\left(\lambda_{p}^{F} g\right) .
$$

Since $\mathcal{S}$ is an isomorphism, $T_{p^{2}} g=\lambda_{p}^{F} g$ for all primes $p$ and thus $g$ equals $f_{K}$ up to a nonzero scalar. Consider the field $K_{f_{K}}=\mathbb{Q}\left(\mu_{2},\left\{\lambda_{p}^{F}\right\}_{p \neq 2}\right)$ where $\mu_{2}$ is the Hecke eigenvalue of $f_{K}$ under the Hecke operator $T_{4}^{+}$. By [3], $\mu_{2}$ is algebraic and hence $K_{f_{K}}$ is a number field.

By [4], it follows that by multiplying $f_{K}$ with $a_{K}\left(\left|D_{0}\right|\right)^{-1}$ where $D_{0}$ is a suitable fundamental discriminant with $(-1)^{\frac{k-1}{2}} D_{0}>0$ and $a_{K}\left(\left|D_{0}\right|\right) \neq 0$ one can assume that the Fourier coefficients of $f_{K}$ lie in $K_{f_{K}}$. Hence the Fourier coefficients of $f_{K}$ are algebraic up to a normalization.

By the above proposition, we can now choose the basis element $f_{1}$ to be equal to $f_{K}$. By the argument as in Theorem 3.2, the following result holds for $S_{k / 2}(N, \chi, F)$.

Corollary 4.3. Let $f=\sum_{n=1}^{\infty} a_{f}(n) q^{n}$ be an element of $S_{k / 2}(N, \chi, F)=\left\langle f_{1}, f_{2}\right\rangle$, where $f_{i}(z):=\sum_{n=1}^{\infty} a_{i}(n) q^{n}$, with $a_{i}(*)$ 's are algebraic. Choose $m_{0} \in \mathbb{N}$ with $m_{0} \not \equiv 0,(-1)^{\frac{k-1}{2}}$ $(\bmod 4)$ such that $a_{1}\left(m_{0}\right)=0$ and $a_{2}\left(m_{0}\right) \neq 0$. Choose $n_{0} \in \mathbb{N}$ such that $a_{1}\left(n_{0}\right) \neq 0$.

If $a_{f}\left(m_{0}\right), a_{f}\left(n_{0}\right)$ are algebraic, then $a_{f}(n)$ are algebraic for all $n \in \mathbb{N}$. Moreover, there exists a number field $K_{f}$ such that $a_{f}(n) \in K_{f}$ for all $n$.

Remark 4.4. One can always find such an $m_{0}, n_{0}$ as in the above Corollary, since $f_{2} \notin S_{k / 2}^{+}(N, \chi, F)$ and $f_{1} \neq 0$.

We illustrate the results in this section with a few examples.

Example 2. Let $F$ be the newform in $S_{2}^{\text {new }}(91)$ given by the following Fourier expansion

$$
F=q-2 q^{2}+2 q^{4}-3 q^{5}-q^{7}-3 q^{9}+6 q^{10}-6 q^{11}+O\left(q^{12}\right) .
$$

We note that $F$ is the newform corresponding to the rank 1 elliptic curve defined by $y^{2}+y=x^{3}+x$. By [6, Corollary 5.2], the space $S_{3 / 2}\left(364, \chi_{\text {triv }}, F\right)$ is generated by

$$
\begin{aligned}
& f_{1}=q^{3}-q^{12}+q^{35}-q^{40}+O\left(q^{50}\right) \\
& f_{2}=q^{10}+q^{12}+q^{13}-q^{14}+q^{17}+q^{26}-3 q^{38}-q^{40}-2 q^{42}-2 q^{48}+O\left(q^{50}\right) .
\end{aligned}
$$

Here $f_{1}$ belongs to Kohnen's +-subspace and where as $f_{2}$ does not. Thus to check whether a given eigenform in $S_{3 / 2}\left(364, \chi_{\text {triv }}, F\right)$ has algebraic Fourier coefficients, we need to take $m_{0}=10$ and $n_{0}=3$ in Corollary 4.3 .

Example 3. Let $G$ be the newform in $S_{4}^{\text {new }}(13)$ given by

$$
G=q+b q^{2}+(-3 b+4) q^{3}+(b-4) q^{4}+(b-2) q^{5}+O\left(q^{6}\right),
$$


where the minimal polynomial of $b$ is $x^{2}-x-4$. Let $\chi_{13}$ be the quadratic Dirichlet character given by the Kronecker symbol $\left(\frac{13}{.}\right)$. By [6, Corollary 5.2], the space $S_{5 / 2}\left(52, \chi_{13}, G\right)$ is generated by

$$
\begin{aligned}
& f_{1}=q+(b+2) q^{4}+1 / 2(-5 b-8) q^{5}+O\left(q^{6}\right) \\
& f_{2}=q^{2}+1 / 19(-2 b+14) q^{3}+(-b+1) q^{4}+1 / 19(18 b+26) q^{5}+O\left(q^{6}\right) .
\end{aligned}
$$

Here $f_{1}$ belongs to Kohnen's + -subspace while $f_{2}$ does not. Thus for a given eigenform in $S_{5 / 2}\left(52, \chi_{13}, F\right)$ to have algebraic Fourier coefficients, we just need to take $m_{0}=2$ and $n_{0}=1$ in Corollary 4.3 .

\section{Sturm's Bound for modular forms in Kohnen's +-Subspace}

In this section, we will give a necessary and sufficient condition for a half-integral weight modular form to be in Kohnen's +-subspace by considering only finitely many terms.

Proposition 5.1. Suppose $f=\sum_{n=1}^{\infty} a_{f}(n) q^{n} \in S_{k / 2}(N, \chi)$. Then, $f \in S_{k / 2}^{+}(N, \chi)$ if and only if $a_{f}(n)=0$ for all $n \equiv 2,(-1)^{\frac{k+1}{2}}(\bmod 4)$ with $1 \leq n \leq B_{k}^{\prime}(N)$, where $B_{k}^{\prime}(N)=\frac{k}{24} \cdot\left[\mathrm{SL}_{2}(\mathbb{Z}): \Gamma_{0}(4 N)\right]$.

Proof. For simplicity, let $B$ denote $B_{k}^{\prime}(N)$. The necessary condition is clear, by definition.

Suppose that $a_{f}(n)=0$ for all $1 \leq n \leq B$ with $n \equiv 2,(-1)^{\frac{k+1}{2}}(\bmod 4)$. Since $a_{f}(n)=0$ for all $1 \leq n \leq B$ with $n \equiv(-1)^{\frac{k+1}{2}}(\bmod 4)$, Sturm's bound for the Fourier coefficients of half-integral weight forms in arithmetic progressions [8, Section 7] implies that $a_{f}(n)=0$ for all $n$ with $n \equiv(-1)^{\frac{k+1}{2}}(\bmod 4)$.

Now, it is enough show that $a_{f}(n)=0$ for all $1 \leq n \leq B$ with $n \equiv 2(\bmod 4)$ implies that $a_{f}(n)=0$ for all $n \in \mathbb{N}$ with $n \equiv 2(\bmod 4)$. Unfortunately we cannot apply the above result in this situation because $(2,4) \neq 1$. However, we can get around to prove the required statement.

Applying $V$ and $U$ operators to $f$ (cf. [9]), we obtain that

$$
V(4) \circ U(4) f(z)=\sum_{n=1}^{\infty} a_{f}(4 n) q^{4 n} \in S_{k / 2}(4 N, \chi),
$$

and

Take

$$
V(2) \circ U(2) f(z)=\sum_{n=1}^{\infty} a_{f}(2 n) q^{2 n} \in S_{k / 2}(2 N, \chi)
$$

$$
g(z)=V(2) \circ U(2) f(z)-V(4) \circ U(4) f(z)=\sum_{n=1}^{\infty} a_{f}(4 n-2) q^{4 n-2} \in S_{k / 2}(4 N, \chi) .
$$

Since $a_{f}(n)=0$ for all $1 \leq n \leq B$ with $n \equiv 2(\bmod 4)$, we see that the first $B$ coefficients of $g(z)$ are zero. By Lemma 3.1 we obtain that $g(z)=0$. Hence $a_{f}(n)=0$ for all $n \equiv 2$ $(\bmod 4)$. This proves the proposition.

It follows from the above proposition that in Corollary 4.3, we can choose $m_{0}, n_{0} \leq$ $B_{k}^{\prime}(N)$.

\section{Acknowledgements}

We thank Prof. W. Kohnen for his encouragement and Prof. G. Wiese for some useful discussions. This work was initiated during the first author's visit to Hausdorff Research Institute for Mathematics, Bonn, and second author's visit to Max Planck Institute for Mathematics, Bonn, during Jan-Feb 2013. We thank these institutes for their hospitalities and also for providing perfect working conditions. 


\section{REFERENCES}

[1] Basmaji, Jacques. Ein Algorithmus zur Berechnung von Hecke-Operatoren und Anwendungen auf modulare Kurven. Ph. D. Dissertation, Universität Gesamthochschule Essen, März 1996.

[2] Bosma, Wieb; Cannon, John and Playoust, Catherine. The Magma Algebra System I: The User Language. J. Symb. Comp. 24 (1997), 235-265. (See also http://magma.maths.usyd.edu.au/magma/)

[3] Kohnen, Winfried. Newforms of half-integral weight. J. Reine Angew. Math. 333 (1982), 32-72.

[4] Kohnen, Winfried. On Hecke eigenforms of half-integral weight. Math. Ann. 293 (1992), no. 3, 427431.

[5] Kohnen, Winfried and Mason, Geoffrey. On the canonical decomposition of generalized modular functions. Proc. Amer. Math. Soc. 140 (2012), no. 4, 1125-1132.

[6] Purkait, Soma. On Shimura's Decomposition. Int. J. Number Theory 9 (2013), no. 6, 1431-1445.

[7] Purkait, Soma. Hecke operators in half-integral weight. To appear in Journal de Thorie des Nombres de Bordeaux.

[8] Purkait, Soma. Explicit applications of Waldspurger's Theorem. LMS J. Comput. Math. 16 (2013), 418-435.

[9] Shimura, Goro. On Modular forms of half integral weight. Ann. of Math. (2) 97 (1973), 440-481.

[10] Sturm, Jacob. On the congruence of modular forms. Number theory (New York, 1984-1985), 275-280, Lecture Notes in Math., 1240, Springer, Berlin, 1987.

[11] Waldspurger, Jean-Loup. Sur les coefficients de Fourier des formes modulaires de poids demi-entier. J. Math. Pures Appl. (9) 60 (1981), no. 4, 375-484.

E-mail address: narasimha.kumar@iith.ac.in

Department of Mathematics, Indian Institute of Technology Hyderabad, Ordnance Factory Estate, Yeddumailaram 502205, Andhra Pradesh, INDiA.

E-mail address: Soma.Purkait@warwick.ac.uk

Mathematics Institute, University of Warwick, Coventry, CV4 7AL, United Kingdom. 